\title{
Farklı Mesafeli ve Üçlü-Çapraz Yerleştirilmiş Çatısız Binalar Etrafındaki Akış Özelliklerinin Deneysel Olarak İncelenmesi
}

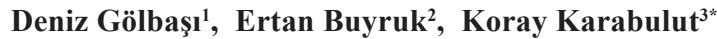

\section{ÖZ}

Çalışmada, üçlü-çapraz yerleştirilmiş ve binalar arası mesafenin $7.5 \mathrm{~cm}$ ve $10 \mathrm{~cm}$ olduğu $5 \mathrm{cmx} 5 \mathrm{cmx} 5 \mathrm{~cm}$ boyutlarında çatısız binalar etrafındaki akış yapıları deneysel olarak Parçacık Görüntülemeli Hız Ölçüm (PIV) tekniği kullanılarak incelenmiştir. Ayrıca, çatısız bir binanın etrafındaki akış analizi daimi, üç boyutlu, k-E türbülans modelli ANSYS Fluent programıyla sayısal olarak yapılmıș ve ulaşılan sonuçlar deneysel sonuçlarla karşılaştırılmıştır. Çalışmada, akım çizgisi $\langle\psi\rangle$ ve hız vektörleri $\langle\mathrm{V}\rangle$ dağılımları çizilmiş ve ortalama eşdeğer hız eğrileri faklı binalar arası mesafeler için araştırılmıştır. $7.5 \mathrm{~cm}$ ' lik mesafeye göre, 10 cm' lik mesafede jet akış bölgesinin oldukça genişlediği ve çapraz olarak yerleştirilen bina çevresindeki ayrılmış akış bölgesinin küçüldüğü belirlenmiştir. Bununla birlikte, deneysel ve sayısal sonuçlar arasında en fazla \% 3' lük bir farklılık bulunmuştur.

Anahtar Kelimeler: Bina aerodinamiği, parçacık görüntülemeli hız ölçümü (PIV), girdap

\section{Experimental Research of Flow Characteristics Around Buildings without Roof Placed Different Distance and Triple-Diagonal}

\begin{abstract}
In study, flow structures around buildings placed triple-diagonal and $7.5 \mathrm{~cm}$ and $10 \mathrm{~cm}$ distances between buildings with dimensions of $5 \mathrm{~cm} \times 5 \mathrm{~cm} \times 5 \mathrm{~cm}$ without roof were investigated as experimental by using Particle Image Velocimetry (PIV) technique. Besides, flow analysis vicinity one building without roof as numerical with ANSYS Fluent program having steady, three dimensional, $\mathrm{k}-\varepsilon$ turbulence model was done and the attained results were matched with these of experimental. In the work, distributions of streamline $\langle\psi\rangle$ and velocity vectors $\langle\mathrm{V}\rangle$ were drawn and average equivalent velocity curves were researched for different distances between buildings. It was determined that jet flow region fairly enlarged at $10 \mathrm{~cm}$ distance according to distance of $7.5 \mathrm{~cm}$ and flow separation area around building placed as diagonal shrank. However, it was found that there is at most a 3\% differences between experimental and numerical results.
\end{abstract}

Keywords: Building aerodynamic, particle image velocimetry (PIV), vortex

\begin{tabular}{|c|c|}
\hline * Iletişism Yazarı & \\
\hline Geliş/Received & $: 08.0$ \\
\hline Kabul/Accepted & : 10.03 .20 \\
\hline
\end{tabular}

1 Dr. Öğr. Üyesi., Sivas Cumhuriyet Üniversitesi, Mühendislik Fakültesi, Makine Mühendisliği Bölümü, Sivas dgolbasi@cumhuriyet.edu.tr, ORCID: 0000-0003-0080-9996

2 Prof. Dr., Sivas Cumhuriyet Üniversitesi, Mühendislik Fakültesi, Makine Mühendisliği Bölümü, Sivas buyruk@cumhuriyet.edu.tr, ORCID: 0000-0002-6539-7614

3 Doç. Dr., Sivas Cumhuriyet Üniversitesi, Sivas Teknik Bilimler Meslek Yüksekokulu, Elek. ve En. Böl.Doğalgaz ve Tesisatı Teknolojisi Prog., Sivas, kkarabulut@cumhuriyet.edu.tr, ORCID: 0000-0001-5680-0988 


\section{EXTENDED ABSTRACT}

\section{Introduction/Backround}

Aerodynamic is an important factor in interaction of noxious aerosols, gases and particles emitted into the atmosphere with terrain, vegetation and buildings by affecting flow structure around buildings. Separation zones and various vortex groups formed by wind interaction between the building and the surrounding space are of great importance in determining the wind interactions affecting buildings and considering the appropriate design parameters. Vibrations caused by the growth of vortexes caused by the interaction of wind with buildings can cause noise and damage in particular.

\section{Objectives/Research Purpose}

The structures and wind interactions around them shape separation zones and multiple vortex systems. The separation zones and vortexes that are formed are of great importance in determining the wind forces acting on buildings. In addition, knowing the flow structures in the buildings contributes to the prevention of particles such as dust, soil, leaves, rain and snow at the ground level from rising with the vortex movements and mixing into the interior environment of the building. Therefore, in this study, flow structures around buildings placed triple and diagonal and $7.5 \mathrm{~cm}$ and $10 \mathrm{~cm}$ distances between buildings with dimensions of $5 \mathrm{~cm} \times 5 \mathrm{~cm} \times 5 \mathrm{~cm}$ without roof were investigated as experimental by using Particle Image Velocimetry (PIV) technique. Besides, flow analysis around one building with dimensions of $5 \mathrm{~cm} \times 5 \mathrm{~cm} \times 5 \mathrm{~cm}$ without roof as numerical with three dimensional, steady, $\mathrm{k}-\varepsilon$ turbulence model by employing ANSYS Fluent program and the attained results were matched with these of experimental.

\section{Methods/Methodology}

In general, PIV technique consists of two steps: visualizing velocity measurement and image processing. Small particles that follow the flow are added to the flow area. These added particles are illuminated twice in a short time with a light source on the surface to be measured. The time difference between the successive illumination of the light source is adjusted according to the average flow rate and magnification scale of the imaging. During the time between the two illuminations, the particles move at the local flow rate. The light scattered by the particles is detected by a high resolution camera placed perpendicular to the light layer and recorded on two frames. The resulting photographic PIV records are then digitized using a scanner and transferred to the computer.

\section{Results/Findings}

In the work, distributions of streamline $\langle\psi\rangle$ and velocity vectors $\langle\mathrm{V}\rangle$ were drawn. Average equivalent velocity curves at $\mathrm{x}$ and $\mathrm{y}$ directions were also analyzed for different distances between buildings. Jet flow region fairly enlarged in the case of $10 \mathrm{~cm}$ distance when matched with the case of $7.5 \mathrm{~cm}$ distance between buildings and it was determined that flow separation area around building placed as diagonal shrank. Also, in this situation jet flow area enlarged and therefore different vortex structures occurred in the rear areas of the building. As a result, it was determined that the flow structure around the buildings and the wind forces acting on the buildings were influenced by the direction and intensity of the incoming wind, as well as the layout of the buildings and the distance between buildings. However, it was determined that there is at most a $3 \%$ deviation between experimental and numerical results.

\section{Discussion and Conclusions}

When the vortexes at the sides and behind the buildings are examined for the distance between both buildings, it is seen that they are not symmetrical to each other. This situation is caused by the reverse flow regions formed in front of the buildings, along the side walls and at the rear as the incoming flow is separated from the corners and edges of the buildings. As a result of this situation, there are decreases in vector densities in the parts where there are vortexes, which proves that the flow velocity decreases here. Therefore, when positioning buildings, they should be designed taking into account the wind flow around them. Besides, turbulence magnitudes increase in the mixing zone separating the reverse and free flow zones. This situation increases the wind forces acting on the buildings. As a result, it is seen that the flow structure around the buildings and the wind forces acting on the buildings are influenced by the direction and intensity of the incoming wind, as well as the placement arrangements of the buildings and the distance between buildings. 


\section{GÍRİŞ}

Aerodinamik, insanlar için sağlıklı ve konforlu bir çevre yaşamı oluşturulmasında ortamın 1sıl şartlarını değiştiren rüzgarı hesaba katan bir iklim unsurudur. Çeşitli büyüklüklerdeki binalar ve baca yapıları, köprüler gibi birçok mühendislik uygulamaları üzerindeki akışlar araştırmacılar tarafından önemli olması sebebiyle özel bir inceleme konusu haline gelmiştir. Bina ve çevre mahalli arasında rüzgar dolayısıyla meydana gelen ayrılma bölgeleri ve çeşitli girdap oluşumları binalar üzerinde etkili olan rüzgar sistemlerinin belirlenmesinde ve en uygun tasarım değişkenlerinin dikkate alınmasında oldukça büyük bir öneme sahiptir. Rüzgarın binalar üzerindeki etkisiyle oluşan girdapların büyümesiyle meydana gelen titreşimler gürültü ve hasara sebebiyet verebilmektedir [1]. Tutar ve Oğuz [2] tek ve çift olarak yerleştirilmiş kare şeklindeki bina yapıları etrafındaki türbülanslı akış bölgelerini farklı rüzgar açıları ve farklı geometrik düzenlemeler için sonlu hacim yöntemiyle incelemişlerdir. RNG türbülanslı model için bulunan sonuçları rüzgar tüneli sonuçları ile kıyaslamışlardır. Sayısal ve deney sonuçlar arasındaki uyumun tek bina düzenlemesi için özellikle bina çatısı civarında olduğunu belirlemişlerdir.

Blocken ve arkadaşları [3] Montreal şehir merkezinde belirledikleri bina etrafindaki kirlilik durumunu rüzgar tüneli deneyleriyle 2 farklı türbülans modelini kullanarak araştırmışlardır. Rüzgâr tüneli sonuçlarını CFD ile doğrulamışlardır. RANS ve LES türbülans modelleri kullanılarak çalışmalar yapılmış ve LES yaklaşımının her iki rüzgar yönü için CFD ve deneysel sonuçlarla uyum içerisinde olduğu tespit edilmiştir. Gölbaşı ve arkadaşları [4] çatısız $5 \times 5 \times 5 \mathrm{~cm}$ ve 30 o eğimli çatı1 $15 \times 5 \times 5 \mathrm{~cm}$ boyutlarında binalar etrafındaki akış yapılarını aralarında $13.75 \mathrm{~cm}$ boşluk olacak şekilde ikili olarak yerleştirilmesiyle Parçacık Görüntülemeli Hız (PIV) tekniğini kullanarak deneysel olarak incelemişlerdir. Gölbaşı ve arkadaşları [5] yaptıkları bir diğer çalışmada, farklı yüksekliklere sahip iki bina modeli civarındaki akış özelliklerini üst görünüş olarak iki farklı ölçüm yüzeyi $(\mathrm{y} / \mathrm{h}=0.5 \mathrm{ve} \mathrm{y} / \mathrm{h}=1)$ ve yan görünüş olarak ise $\mathrm{y} / \mathrm{h}=0.5$ yüzeyi için parçacık görüntülemeli hız ölçüm tekniğini kullanarak deneysel olarak araştırmışlardır. Çalışmalarında ayrıca, sonlu hacimler yöntemi ANSYS Fluent programıyla sayısal olarak da incelemişlerdir. Binaların köşelerinden ve kenarlarından gelen rüzgarların ayrılmasının bir sonucu olarak çatı yüzeylerinde, binaların yan kenarları boyunca ön ve arkalarında ters akış bölgeleri oluştuğunu belirlemişlerdir. Daemei ve Eghbali [6], yüksek binaların türbülanslı bölgesinin uzunluğunu azaltmak için yuvarlatılmış köşeler, oluklu köşeler ve girintili köşeler dahil olmak üzere aerodinamik modifikasyonlar yoluyla kare ve üçgen formlarında aerodinamik davranışın analizini gerçekleştirmişlerdir. 16 bina modeli üzerinde gerçekleştirilen rüzgar tüneli sayısal simülasyonuna benzer şekilde Autodesk akış tasarımı yazılımını kullanmışlardır. Yaklaşık 150 m yüksekliğe sahip 50 katlı yüksek binalar tasarlamak için üçgen şekle sahip modelin rüzgar yönüne bakan oluklu köşesinin aerodinamik modifikasyonu ile 
diğer modellere kıyasla en iyi aerodinamik davranışa sahip olduğunu saptamışlardır. Wheatley ve arkadaşları [7] kat kat inşa etme ilkeleri olan origami ve kirigamiyi kullanarak tasarlanmış dinamik bina yapılarını dikkate alarak şiddetli rüzgarlı darbeleri sönümlemek için daha kapsamlı bir yapı tasarımını gerçekleştirmişlerdir. Rüzgar akışına bir açıda yönlerin sayısını artırarak ve bina cephelerinin boyutunu azaltarak, rüzgar akışına karşı doğrudan gövde boyutunun en aza indirilebileceğini ve rüzgar direncinin azaltılabileceğini belirlemişlerdir. Yan ve Li [8] aerodinamik düzenleme ile ikiz yüksek binalar arasındaki etkileşimin sonuçlarını incelemek amacıyla rüzgar tünelinde deneyler yapmışlardır. Çalışmada, rüzgarın oluşturduğu tepkiyi, yerel basınç katsayılarını ve küresel aerodinamik yüklerle etkileşimin etkilerinin bir bütün olarak değerlendirilebilmesi için kontur çizimini sunmuşlardır. Sonuçlar, dinamik rüzgar yüklerinin ve tepkilerinin, ikiz kulelerin sıralı ve çapraz düzenlemelerinde önemli ölçüde arttığını ve en az değerdeki negatif basınç katsayısı değerlerinin, etkileşimli olmayanlardan yaklaşık olarak \%30 daha büyük olduğunu kanıtlamıştır.

Yapılar ve etraflarındaki rüzgar etkileşimleri, ayrılma bölgelerini ve çoklu girdap sistemlerini şekillendirmektedir. Oluşan ayrılma bölgeleri ve girdaplar, binalara etkiyen rüzgar kuvvetlerini belirlemede büyük bir öneme sahiptir. Ayrıca, binalardaki akış yapılarının bilinmesi hem tasarım açısından hem de zemin seviyesinde bulunan toz, toprak, yaprak, yağmur ve kar gibi parçacıkların girdap hareketleri ile yükselerek bina iç ortamına karışmasının engellenmesine katkı sağlamaktadır. Bu nedenle, bu çalışmada bina merkezleri arası mesafenin $7.5 \mathrm{~cm}$ ve $10 \mathrm{~cm}$ olduğu $5 \mathrm{~cm} \times 5 \mathrm{~cm} x 5 \mathrm{~cm}$ boyutlarında çapraz olarak konumlandırılmış üç adet çatısız bina modelinin etrafındaki akış yapıları deneysel olarak Parçacık Görüntülemeli Hız tekniği (PIV) kullanılarak incelenmiştir. Bunun yanı sıra, çatısız $5 \mathrm{~cm} \times 5 \mathrm{~cm} \times 5 \mathrm{~cm}$ boyutlarındaki bir binanın etrafındaki akış analizi sayısal olarak üç boyutlu, zamandan bağımsız, k- $\varepsilon$ türbülans modelli ANSYS Fluent programından yararlanılarak yapılmış ve ulaşılan sayısal sonuçlar deneysel sonuçlarla kıyaslanmıştır. Başlangıçta deneylerde anlık hız alanlarına ulaşılmış ve sonra bu veriler kullanılarak akım çizgisi $\langle\psi\rangle$ ve hız vektörleri $\langle$ V $>$ dağılımları çizilmiştir. Bununla birlikte, ayrıca ortalama eşdeğer hız eğrileri dağılımları her iki binalar arası mesafe için analiz edilmiştir.

\section{DENEYSEL YÖNTEM}

Bu çalışmada gerçekleştirilen deneyler, kapalı devre olarak işlem gören açık yüzeyli bir su kanalında Parçacık Görüntülemeli Hız Ölçme Tekniğiyle yapılmıştır. Kanal malzemesi olarak fiberglas malzeme kullanılmıştır. Kanal, iki adet su tankı ve tanklar arasına yerleştirilen test kanalı adı verilen şeffaf akrilik bir parçadan meydana gelmektedir.

Test kanalı 750 mm yüksekliğe, 1000 mm genişliğe ve 9000 mm uzunluğa sahiptir. Suyun su depoları arasındaki dolaşımı $15 \mathrm{~kW}$ gücünde santrifüj bir pompa ile sağlan- 
maktadır. Test bölgesinde farklı hızlarda deneyler gerçekleştirebilmek için santrifüj pompanın periyodu frekans kontrolü olan hız ünitesi vasıtasıyla ayarlanabilmektedir. Pompa, çıkıştaki su tankından suyu emip, girişteki su tankına göndermektedir. Deneyler sırasında, test kanalında akışın sürekliliğini temin edebilmek için girişteki tankın çıkışına akış düzenleyici bir bal peteği biçiminde levha yerleştirilmiş ve giriş rezervuarının çıkışı ve test kanalının birleşimi arası oran olarak 2:1 daraltılarak bağlantı yapılmıştır. Test sisteminde giriş deposu aynı zamanda su bekleme odası olarak da hizmet vermekte ve buradan su akış düzenleme bölümüne geçerek test kanalına girmektedir. Sistemde suyu tank içerisindeki yabancı parçacık ve kirlerden ayıran filtrasyon sistemi bulunmaktadır. Bununla birlikte, su kanalı ile parçacık dolaşım sistemlerinin 1 sıl temasını minumum düzeyde tutabilmek amacıyla laboratuvar $22^{\circ} \mathrm{C}$ ' lik bir oda sıcaklığında tutulmuştur. Bunun yanı sıra, lazer 1şınını güneş 1şı̆̆ından korumak için laboratuvar camları özel perdelerle kapatılmıştır. Test kanalının görünüşü Şekil 1 'de gösterilmektedir.

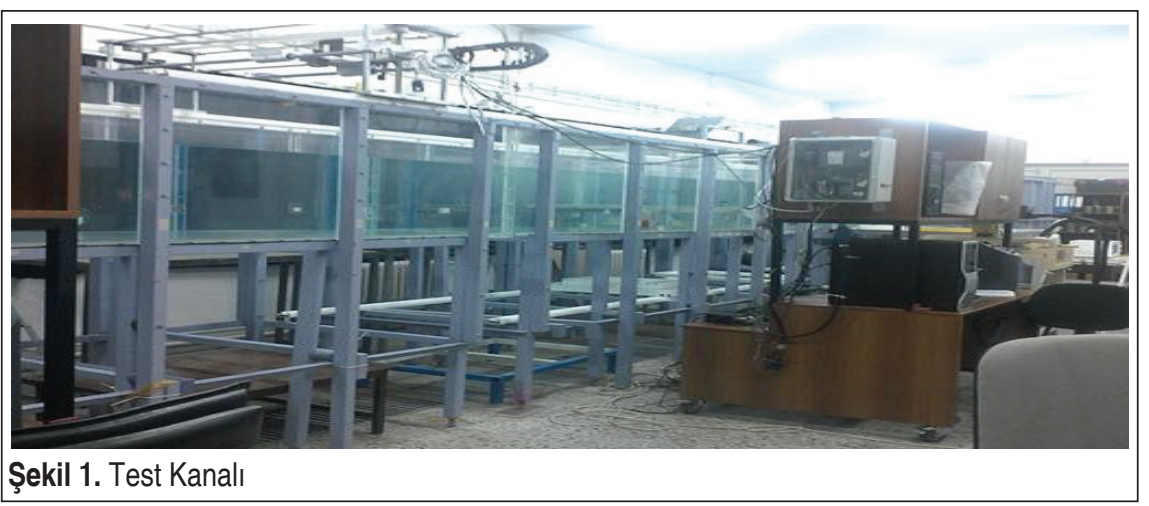

Genel olarak, parçacık görüntülemeli hız ölçüm tekniği iki adımdan oluşur: hız ölçümünü görselleştirme ve görüntü işleme. Akışı takip edebilmek amacıyla akış alanına küçük boyutlu parçacıklar katılmaktadır. Katılan parçacıklar, ölçülecek yüzeydeki bir 1şık kaynağı ile kısa sürede iki kez aydınlatılır. Işık kaynağının ardışık aydınlatması (çoğunlukla lazer) arasında geçen zamansal fark, görüntülemenin ortalama akış hızına ve büyütme ölçeğine göre ayarlanır. Parçacıklar, iki aydınlatma arası geçen süre itibariyle yerel akış hızında hareket ederler. Parçacıkların saçtığ 1 1şık, 1ş1k katmanına dik yerleştirilen yüksek çözünürlüklü kamera ile algılanır ve iki kare üzerine kaydedilir. Elde edilen fotoğrafik PIV kayıtları daha sonra bir tarayıcı kullanılarak sayısallaştırılır ve bilgisayara aktarılır [9]. Şekil 2(a), PIV' nin çalışma prensibini göstermektedir. Akış özelliklerini araştırmak için şeffaf akrilik malzemeden yapılmış 3 yapı modeli kullanılmıştır. Su kanalı içindeki test alanının şematik görünümü Şekil 2(b)'de verilmiştir. Deneyler sırasında su kanalındaki su yüksekliği sabit $0.45 \mathrm{~m}$ olarak alınmıştır. 


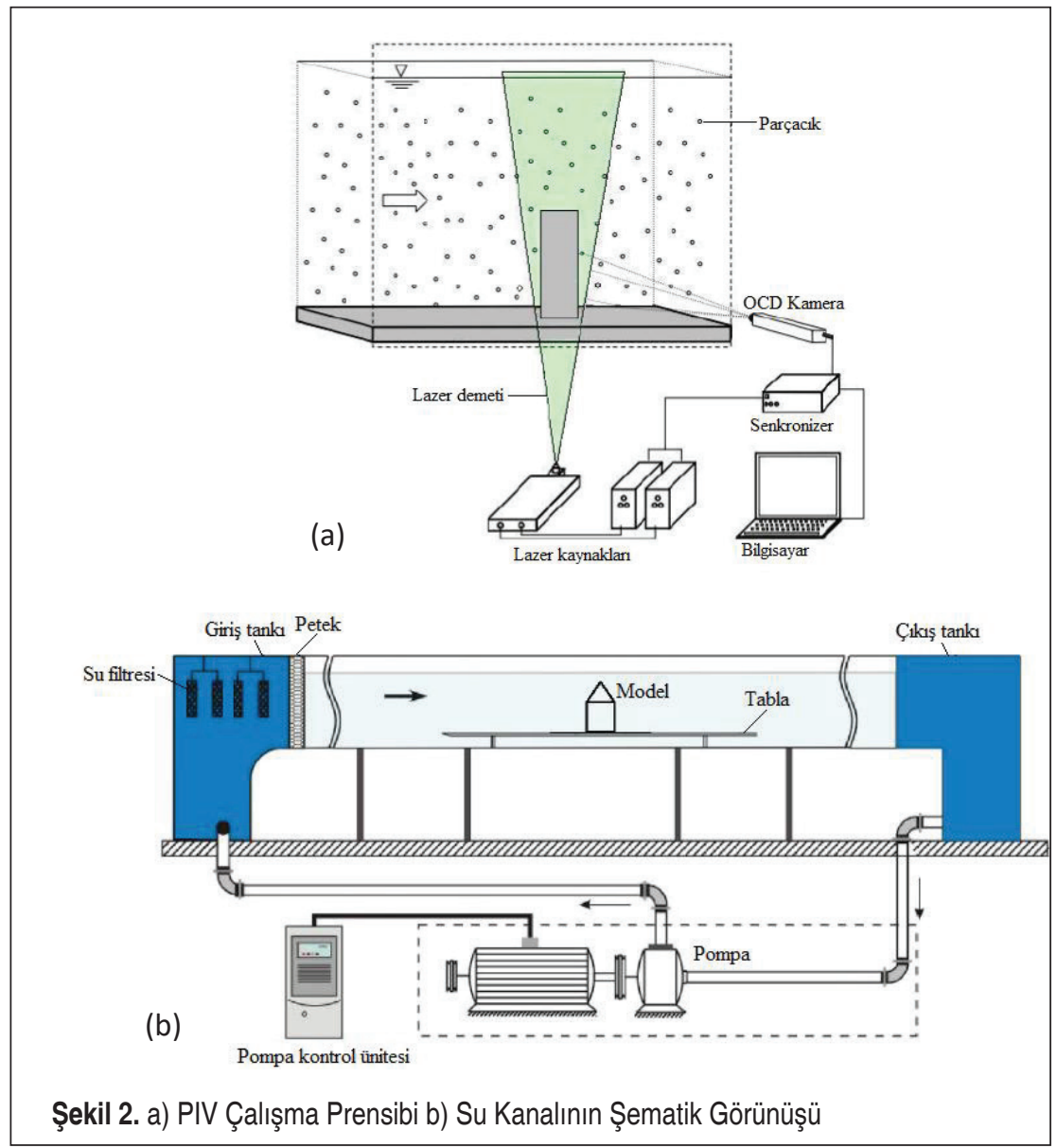

Düzlem platformunun ön kenarı, akışın bozulmasını önlemek için pah kırma ile inceltilmiştir. Model, türbülans etkilerine karşı koruma sağlamak amacıyla kanalın girişinden $1.5 \mathrm{~m}$ uzağa monte edilmiştir. Deneyler, $210 \mathrm{~mm} / \mathrm{s}^{\prime}$ lik bir serbest akış hızında gerçekleştirilmiştir.

\section{3. ÜST GÖRÜNÜŞ ÖLÇÜM YÜZEYLERİ}

Şekil 3(a)' da gösterildiği gibi kamera kanalın karşısına yerleştirildiğinde, lazer, üst görünüş için binanın önüne yerleştirilmekte ve deneysel sistem, kameranın önünde 45o' lik bir eğimle ayna konumlandırılarak oluşturulmaktadır. Deneysel sistemde üst görünüş için kamera ve ayna yerleşimleri Şekil 3(b)' de belirtilmektedir. Bununla birlikte, Şekil 3(c)' de gösterilen simetri yüzeyi, üst görünüş için $y / H=0.5$ ' de alınmiştır. 


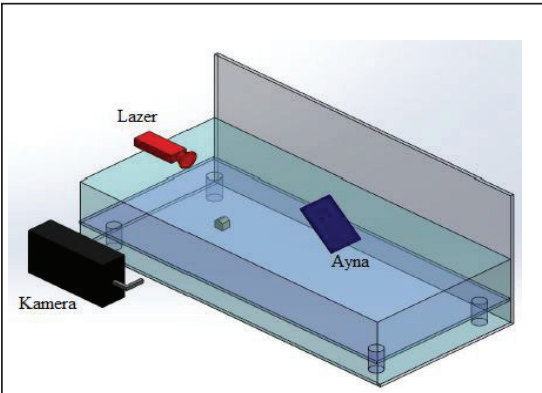

(a)

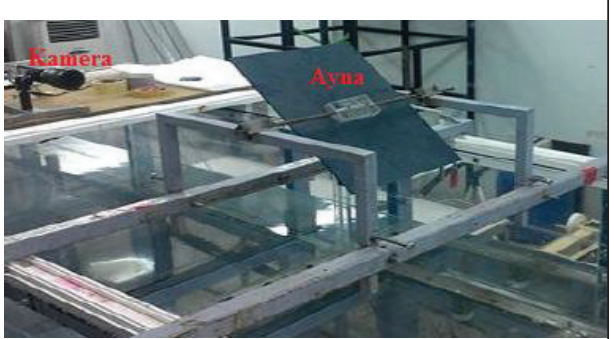

(b)

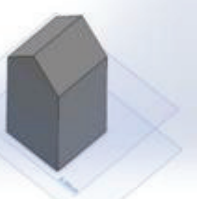

(c)

Şekil 3. a) Üstten Görünüş Için Tertibatın Yerleşimi, b) Deneysel Sistemde Üstten Görünüş Için Kamera ve Aynanın Yerleşimi, c) Üstten Görünüş Ölçüm Yüzeyi

\section{DENEYSEL SONUÇLAR}

Herhangi bir konum ve zamandaki hız, türbülans hız bileşenlerini ve ortalamaların toplamını içermektedir. Bu çalışmada, her bir ölçüm yüzeyi için ortalama hızlar ortalama olarak 500 görüntü için hesaplanmıştır. Akım çizgileri, herhangi bir anda yerel hız vektörlerinin tanjantı olan çizgilerin birleşimi olarak tanımlanmıştır. Bununla birlikte, girdap akış parçacıklarının dönmesinin bir ölçüsüdür. Her bir ölçüm yüzeyi için girdap değerleri, ortalama hızlar kullanılarak hesaplanmıştır.

Deneylerin sonucu olarak, binalar etrafında oluşan akış yapısı akım çizgileri $\langle\psi\rangle$ ve hız dağılımı $<$ V > olarak sunulmuştur. Elde edilen deneysel verilerde, hız vektörleri akış dağılımı ve yönünü belirtirken, akım çizgileri anlık akış verilerinin anlaşılmasını kolaylaştırmaktadır. Ortalama eşdeğer hız eğrileri, türbülans sınır tabakanın etkisiyle

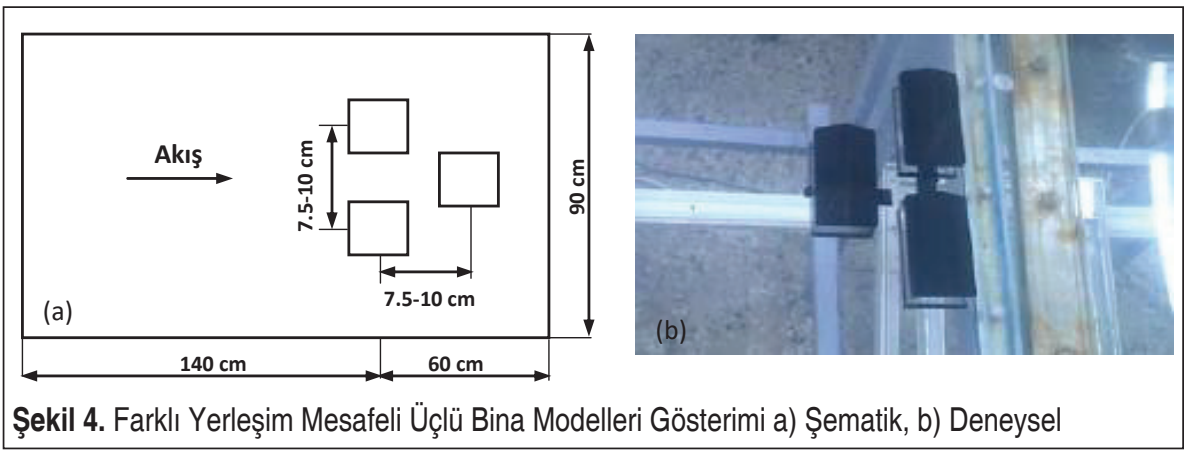


oluşmuştur. Ayrıca, Şekil 4(a) ve (b)' de sırasıyla birbirlerinden farklı mesafelerde yerleştirilmiş binaların şematik ve deneysel konumlanmaları gösterilmektedir.

Şekil 5(a), (b)'de sırasıyla 5cmx 5cmx 5cm boyutlarında üç adet çatısız bina modelinin kanal içerisindeki plaka üzerine bina merkezleri arası mesafenin $7.5 \mathrm{~cm}$ olduğu durum için çapraz olarak yerleştirilmesiyle akım çizgileri $\langle\psi\rangle$ ve hız vektörleri $<$ V $>$ dağ1lımları incelenmiştir. Şekil 5(a)' da görüldüğü gibi binaların yan kısımlarında ve bina arkalarındaki girdaplar incelendiğinde birbirlerine simetrik bir yapıda olmadıkları görülmektedir. Bu duruma, gelen akışın binaların köşe ve kenarlarından ayrılmasıyla yapıların önünde ve yan duvarları boyunca ve arka kısmında oluşan ters akış bölgeleri sebep olmaktadır. Bu durumun sonucu olarak da girdapların olduğu kısımlarda akış hızları azalmaktadır. Bununla birlikte, üst bina modelinde bina ön yüzeyinden Sab1 noktasından yukarıya doğru ayrılan akış model sol yan köşesinden ayrılarak düzensiz bir F1 girdap bölgesi meydana getirmektedir. Oluşan girdap merkezinin koordinatları $\mathrm{x}=50.65 \mathrm{~mm}$ ve $\mathrm{z}=179.52 \mathrm{~mm}$ noktasındadır. Aşağıya doğru ayrılan akış, model sağ yan köşesinden ayrılmadan yukarıya doğru akışına devam etmektedir. Bu nedenle yüzey üzerinde ayrılmış akışa sahip bir ikincil akış bölgesi oluşmamaktadır. Üst bina ile alt bina arasında kısa mesafe nedeniyle burada jet akış oluşmaktadır. İki bina arasında oluşan jet akış yüksek hızla arkadaki binaya çarparak $\mathrm{S}_{\mathrm{ab} 2}$ noktasından aşağ1 ve yukarıya doğru ayrılmaktadır. Üst bina arkasındaki akış yapısı incelendiğinde, jet akış etkisiyle binanın sağ yan tarafından yukarı doğru yönlenmiş akışla binanın sol yan tarafından tekrar tutunan akış birleşmektedir. Ayrılan akış kısmından itibaren bir girdap meydana gelmiştir. Bu girdabın merkezi $\mathrm{x}=171.06 \mathrm{~mm}$ ve $\mathrm{z}=148.41 \mathrm{~mm}$ noktasında olup, saat yönünde dönmektedir. Arka binanın alt kısmında $F_{2}$ girdabına simetrik olmayan bir girdap bölgesi oluşmuş ve bu girdap bölgesi bölünerek büyük $\mathrm{F}_{3}$ ve devamında küçük bir $\mathrm{F}_{4}$ girdap bölgesi oluşturmuştur. Büyük $\mathrm{F}_{3}$ girdap merkezinin koordinatları $\mathrm{x}=167 \mathrm{~mm}$ ve $\mathrm{z}=79.92 \mathrm{~mm}$ noktasındadır. Küçük F4 girdabı ise $\mathrm{x}=$ $110.87 \mathrm{~mm}$ ve $\mathrm{z}=59.12 \mathrm{~mm}$ noktasındadır. Simetrik olarak oluşmayan bu iki girdap arasındaki durma noktası olan $\mathrm{S}_{1}$ noktasi $\mathrm{x}=232.98 \mathrm{~mm}$ ve $\mathrm{z}=116.28 \mathrm{~mm}$ olarak belirlenmiştir. Alt tarafa yerleştirilen bina modelinde Sab3 noktasından aşağıya doğru ve yukarıya doğru ayrılan akış, üst taraftaki bina modelinin akış yapısına benzer yapıda olup aşağı ayrılan akış bina sağ yan köşesinden ayrılarak $\mathrm{F}_{5}$ girdap bölgesini oluşturmuştur. Bu girdap merkezinin koordinatları $\mathrm{x}=52.99 \mathrm{~mm}$ ve $\mathrm{z}=45.89 \mathrm{~mm}$ noktasındadır. Şekil 5(b)'de ölçümler sonucu ulaşılan 7326 (99x74) adet vektör gösterilmektedir. Zaman ortalamalı hız alanları $<\mathrm{V}>$ için bina yakınlarındaki hız vektörleri ve hız vektör uzunluklarının da azalması nedeniyle bu bölgelerde hızlar azalmıştır. Bununla birlikte, bina çevresinden uzaklaştıkça hız vektörlerinin bulunduğu alanların arttığı ve hız vektör uzunluklarının artması sebebiyle de hız değerlerinin arttığı görülmektedir. Hızın yükseldiği bölgeler, özelikle bina üzerinde oluşan girdap üst bitim noktalarında bulunmaktadır. 

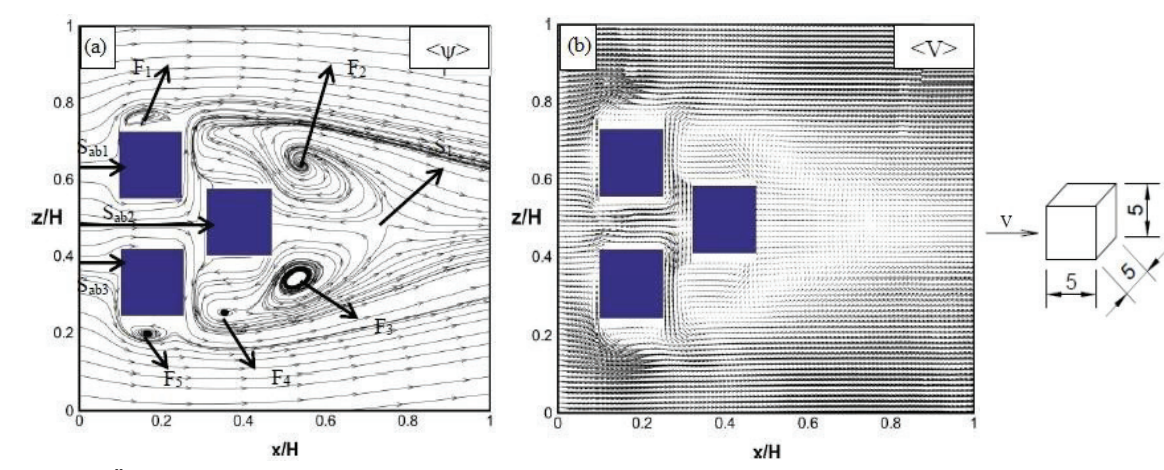

Şekil 5. Üçlü Olarak Yerleştirilmiş Binalar Arası $7.5 \mathrm{~cm}$ Mesafe Olan Bina Modelleri Civarındaki Akış İçin y/H = 0.5 Simetri Yüzeyi Zaman Ortalamalı a) Akım Çizgileri $\langle\psi\rangle$, b) $H ı z$ Dağılımı $<V\rangle$

Üçlü olarak çapraz şekilde yerleştirilen üç adet çatısız model için binalar arası mesafenin $7.5 \mathrm{~cm}$ olması durumunda $\mathrm{x}$ ve y yönlerindeki ortalama eşdeğer hız eğrileri dağılımları sırasıyla Şekil 6(a) ve (b)'de gösterilmektedir. Binalar arasında jet akış bölgesi oluşmuştur ve oluşan bu jet akış bölgesi binalar arası mesafeye bağlı olarak genişleyebilmekte fakat bu durumda hız azalmaktadır. Arka binada oluşan ayrılmış akış bölgesi x/H = 0.65 noktasında olup, binalar arası mesafenin artmasıyla gerileyebilmektedir(Şekil 6(a)). Şekil 6(b)'de y yönündeki ortalama eşdeğer hız dağılımları verilmektedir. Burada, özellikle görüldüğü gibi kanal girişinin alt ve üst kısımlarındaki binaların keskin köşelerinde oluşan akışlar bina arkalarında oluşan akışlardan ayrılmış olup, birbirlerinden bağımsızdırlar.
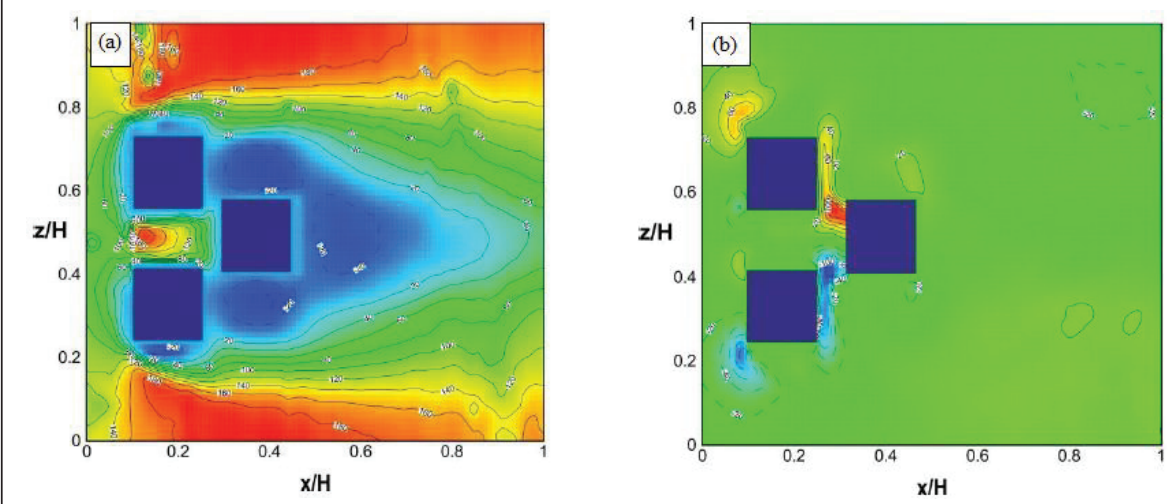

Şekil 6. Üçlü Olarak Yerleștirilen Binalar Arası $7.5 \mathrm{~cm}$ Mesafe Olan Çatısız Bina Modelleri Etrafındaki Akış ıçin y/H=0.5 Simetri Yüzeyi Zaman Ortalamalı a) $<\mathrm{u}>\mathrm{H}$ ız Dağılımı, b) $<v>\mathrm{H}$ zz Dağılımı 
Boyutları $5 \mathrm{~cm} \times 5 \mathrm{~cm} \times 5 \mathrm{~cm}$ ve bina merkezleri arası mesafenin $10 \mathrm{~cm}$ olduğu üç adet çatısız bina modeli için binalar etrafındaki zaman ortalamalı akım çizgileri $\langle\psi\rangle$ ve hız vektörleri $<$ V $>$ dağılımları sırasıyla Şekil 7(a) ve (b)' de verilmektedir. Şekil 7(a)' da görüldüğü gibi binaların yan kısımlarında ve bina arkalarındaki girdaplar incelendiğinde, oluşan ters akış bölgeleri sonucu birbirlerine simetrik bir yapıda olmadıkları dikkati çekmektedir. Bunun yanı sıra, binalar arası mesafenin $7.5 \mathrm{~cm}$ olduğu duruma göre mesafenin artması nedeniyle jet akış alanı genişlemiş ve buna bağlı olarak bina arka bölgelerinde farklı girdap yapıları oluşmuştur. Ayrıca arka kısma yerleştirilmiş binanın yan bölgelerinde oluşan girdap yapıları küçülerek değişime uğramıştır. Üst bina modelinin bina ön yüzeyinde $S_{a b 1}$ noktasından yukarıya doğru ayrılan akış, model sol yan köşesinden ayrılarak bir $\mathrm{F}_{1}$ girdap bölgesi meydana getirmektedir. Oluşan girdap merkezinin koordinatları $\mathrm{x}=47.21 \mathrm{~mm}$ ve $\mathrm{z}=191.873 \mathrm{~mm}$ ' dir. Aşağıya doğru ayrılan akış, model sağ yan köşesinden akışın bir kısmı ayrılmadan yukarıya doğru akışına devam ederken $\mathrm{F}_{1}$ girdabının tekrar birleşme noktasındaki akışla birleşmektedir. Bu noktada $\mathrm{S}_{1}$ durma noktası oluşmuş olup, bu noktanın merkezi $\mathrm{x}=106.25 \mathrm{~mm}$ ve $\mathrm{z}=182.89 \mathrm{~mm}$ olarak belirlenmiştir. S1 durma noktasının sol yanında küçük bir $F_{2}$ girdabı ve altında ona daha büyük bir $F_{3}$ girdabı oluşmuştur. $F_{2}$ girdap merkezinin koordinatları $\mathrm{x}=95.83 \mathrm{~mm}$ ve $\mathrm{y}=186.20 \mathrm{~mm}$ noktasında olup saat yönünde dönmektedir. Bununla birlikte, $F_{3}$ girdap merkezi ise $x=96.41 \mathrm{~mm}$ ve $z=153.13 \mathrm{~mm}$ noktasında olup saat yönünün tersinde dönmektedir. İki bina arasında oluşan jet akış, yüksek bir hızla arkadaki binaya çarparak Sab2 durma noktasından aşağ1 ve yukarı ayrılmıştır. Üst yan ve arka bölgede oluşan girdap bölgeleri eşit boyutlarda bölünerek F4 ve devamında F5 girdap bölgelerini oluşturmuştur. F4 girdabının merkezi $\mathrm{x}=$ $155.44 \mathrm{~mm}$ ve $\mathrm{z}=154.55 \mathrm{~mm}$ noktasında olup saat yönünde dönmektedir. F5 girdab1 ise $\mathrm{x}=198.28 \mathrm{~mm}$ ve $\mathrm{z}=134.71 \mathrm{~mm}$ noktasında olup yine saat yönünde dönmektedir. Alt yan ve arka bölgede oluşan girdap bölgeleri eşit boyutlarda bölünerek $\mathrm{F}_{6}$ ve devamında $\mathrm{F}_{7}$ girdap bölgelerini oluşturmuştur. $\mathrm{F}_{6}$ girdap merkezi $\mathrm{x}=195.96 \mathrm{~mm}$ ve $\mathrm{z}=97.86 \mathrm{~mm}$ noktasında olup saat yönünün tersinde dönmektedir. $\mathrm{F}_{7}$ girdabı ise $\mathrm{x}=$ $153.13 \mathrm{~mm}$ ve $\mathrm{z}=77.07 \mathrm{~mm}$ noktasında olup saat yönünde dönmektedir. Simetrik olarak oluşan bu iki girdap arasındaki durma noktası olan $S_{2}$ noktası $x=243.42 \mathrm{~mm}$ ve $\mathrm{z}=112.58 \mathrm{~mm}$ olarak belirlenmiştir. Alt tarafa yerleştirilen bina modelinde Sab3 noktasından aşağı ve yukarı ayrılan akış, üst taraftaki bina modelinin akış yapısına simetrik oluşmuş olup, yukarı ayrılan akışın bir kısmı ayrılmadan aşağıya doğru hareket etmektedir. Akışın diğer kısmı bina arkasında iki girdap bölgesi oluşturmuştur. Büyük olan $\mathrm{F}_{8}$ girdap merkezi, $\mathrm{F}_{3}$ girdap merkezinin $\mathrm{x}$ koordinatıyla aynı noktada oluşmuş olup $\mathrm{x}=96.41 \mathrm{~mm}$ ve $\mathrm{z}=86.99 \mathrm{~mm}$ noktasındadır ve saat yönünde dönmektedir. Büyük girdabın devamında oluşan $F_{9}$ girdap merkezinin koordinatları $\mathrm{x}=97.56 \mathrm{~mm}$ ve $\mathrm{z}=52.04 \mathrm{~mm}$ noktasındadır. Bu girdabın sağ yanında $\mathrm{S}_{3}$ durma noktası oluşmuş olup $\mathrm{x}=113.77 \mathrm{~mm}$ ve $\mathrm{y}=52.87 \mathrm{~mm}$ olarak belirlenmiştir. Bununla birlikte, bina ön yüzünden aşağı doğru ayrılan akış bina sağ yan köşesinden ayrılarak $\mathrm{F}_{10}$ girdap bölgesini 

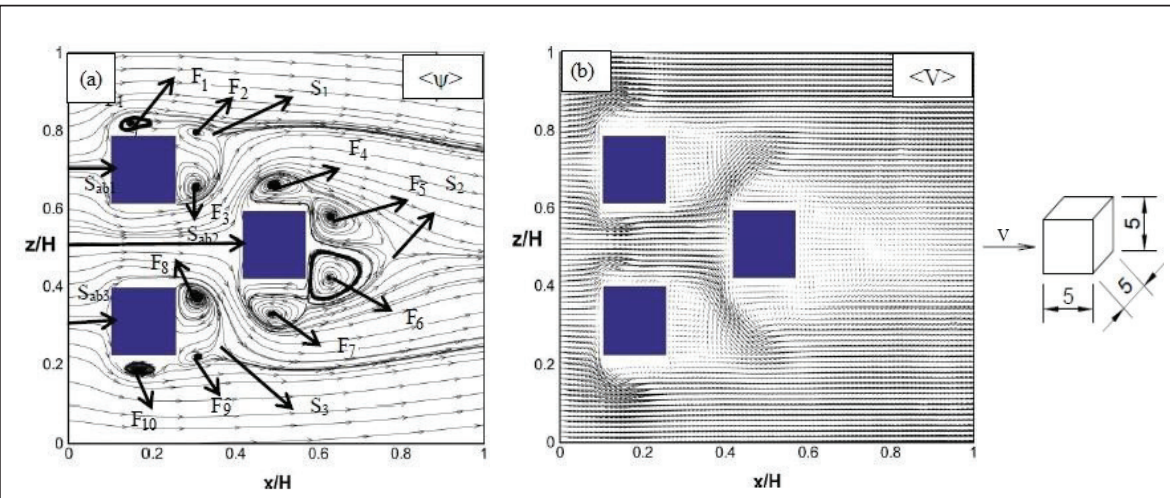

Şekil 7. Üçlü Olarak Yerleştirimiş Binalar Arası $10 \mathrm{~cm}$ Mesafe Olan Bina Modelleri Civarındaki Akış ıçin y/H = 0.5 Simetri Yüzeyi Zaman Ortalamalı a) Akım Çizgileri $\langle\psi\rangle$, b) $H ı z$ Dağılımı $<V\rangle$

oluşturmuştur. Bu girdap merkezi $x=52.99 \mathrm{~mm}$ ve $\mathrm{z}=44 \mathrm{~mm}$ noktasındadır. Ayrıca, Şekil 7(b)'de zaman ortalamalı hız dağılımlarında $<V>$ jet akışın etkisi ile hızın yüksek yoğunluklu olduğu alanlar daha açık bir şekilde görülmektedir.

Üçlü olarak çapraz şekilde yerleştirilen binalar arası mesafenin $10 \mathrm{~cm}$ olduğu $5 \mathrm{~cm} \times 5 \mathrm{~cm} \times 5 \mathrm{~cm}$ boyutlarındaki üç adet çatısız modelin etrafındaki ortalama eşdeğer hız eğrileri dağılımları $y / H=0.5$ simetri yüzeyinde, $x$ ve y yönleri için sırasıyla Şekil 8 (a) ve (b)'de verilmektedir. Binalar arası mesafenin $7.5 \mathrm{~cm}$ olduğu durumla kıyaslandığında (Şekil 8(a)) jet akış bölgesinin oldukça genişlediği ve çapraz olarak yerleştirilen bina çevresindeki ayrılmış akış bölgesinin küçüldüğü görülmektedir. Aynı şekilde, y yönündeki ortalama eşdeğer hız eğrileriyle karşılaştırıldığında yine jet akış bölgesinin genişlediği ve kanal girişindeki alt ve üst kısımlardaki binaların alt ve üst bölgelerinde aşağı ve yukarı akış bölgelerinin oluştuğu saptanmıştır. Ayrıca, çapraz bina ön bölgesindeki aşağı ve yukarı akış bölgesi arası mesafeleri artmıştır.

\section{SAYISAL INCELEME}

Sayısal çalışma, üç boyutlu ve daimi olarak k- $\varepsilon$ türbülans modelli sonlu hacimler yöntemi olan ANSYS Fluent bilgisayar programı kullanılarak yapılmıştır. Çalışmada, tek $5 \mathrm{~cm} \times 5 \mathrm{~cm} \times 5 \mathrm{~cm}$ boyutlu çatısız bina için elde edilen deneysel çalışma sonucunun (PIV), sayısal çalışmadan elde edilen sonuçlarla karşılaştırılması amaçlanmıştır.

Sonlu hacim yöntemi, bölümlerin her biri için bir çözüm bulmak üzere çözülecek geometriyi parçalara ayırma ve daha sonra soruna genel bir çözüm bulmak amaciyla bu çözümleri birleştirmek ilkesine dayanmaktadır. Bu yöntem, 1sı, akış denklemlerini sayısal olarak çözülebilen cebirsel denklemlere dönüştürmek için kontrol hacmine dayalı bir teknik kullanır. Diğer bir deyişle, bu teknik, her bir kontrol hacminde 1sı, 

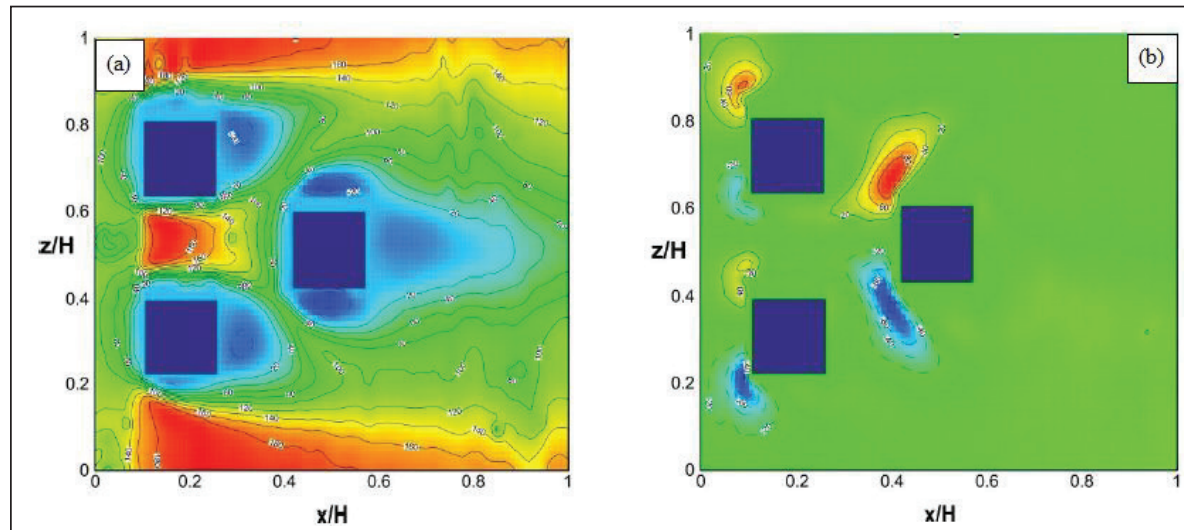

Şekil 8. Üçlü Olarak Yerleştirilen Binalar Arası $10 \mathrm{~cm}$ Mesafe Olan Çatısız Bina Modelleri Etrafındaki Akış ıçin y/H=0.5 Simetri Yüzeyi Zaman Ortalamalı a) $<u>H ı z$ Dağııımı b) $<v>$ hız Dağılımı

akış denklemlerinin integrasyonunun alınması esasına dayanmaktadır. Bu integrasyon sonucu, oluşan her bir kontrol hacmini temsil eden denklemleri sağlar. En uygun ağ modelinin hazırlanması için hız, basınç ve sıcaklık gibi değişkenlerdeki değişimin daha büyük olduğu bölgelerde ince bir ağ yapısı oluşturulmalıdır. Bu nedenle, model binası çevresindeki hacim bölgeleri için en iyi ağ yapısı kullanılmış ve diğer bölgelerde daha seyrek bir ağ tercih edilmiştir. Sayısal çalışmada kullanılan çatısız model, 1.055156 adet eleman içermektedir. Hesaplamaların yakınsaması, süreklilik ve momentum denklemleri için yakınsama ölçeği 10-6‘ dan küçük olduğunda, enerji denklemi için ise 10-7'den küçük olduğunda durdurulmuştur. Simülasyon için üç boyutlu piramitten oluşan ızgara yapısı kullanılmıştır (Şekil 9).

Bununla birlikte, sayısal model deneysel ortam koşulları kullanılarak gerçekleştirilmiştir. $0.193 \mathrm{~m} / \mathrm{s}$ akış hızı durumu için model etrafında üç boyutlu detaylı akış ya-
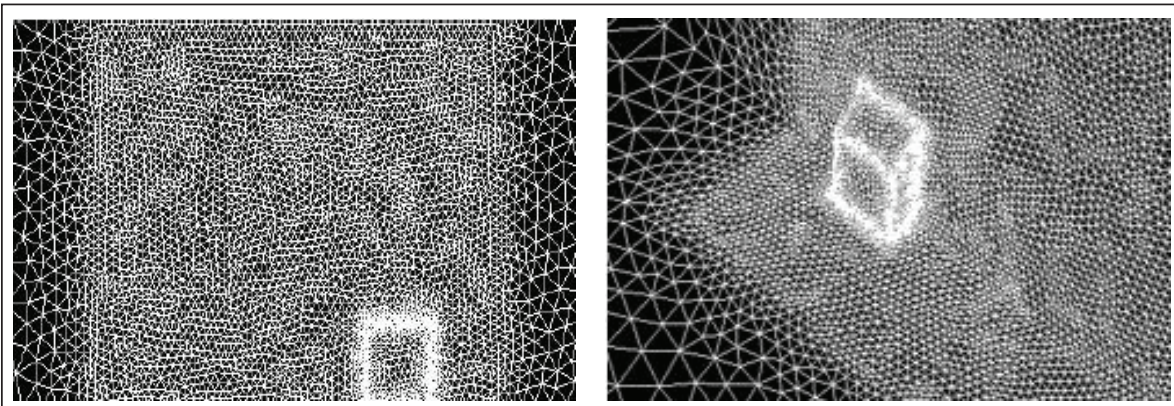

Şekil 9. Sayısal Modellemede Kullanılan Ağ Yapısı 
pıları elde edilmiştir. Su kanalının girişinde ortalama türbülans yoğunluğu oldukça düşük olduğundan (\% 0.5), sayısal çalışmada kanal girişinde düzgün hız profili, uœ uygulanmıştır. Çalışmada, bina çevresinde simetri ve duvar sınır koşulları kullanılmıştır. Kanal akışı için ise çıkış akışı sınır koşulundan yararlanılmıştır. Ayrıca, sayısal çalışmada binaların akışta türbülansa neden olması nedeniyle, aerodinamikte sıklıkla kullanılan k-E türbülans modeli kullanılırken akış alanının kanal için laminer olduğu varsayılmıştır.

Geometri boyunca akış ve 1sı transferi, kütle, momentum ve enerjinin korunumu yasalarından üretilen ve bir gövde kuvveti olmaksızın zamandan bağımsız olarak türetilen ve aşağıdaki gibi ifade edilen kısmi diferansiyel denklemler çözülerek elde edilmiştir ([9], [10], [11]).

Süreklilik denklemi

$\frac{\partial u}{\partial x}+\frac{\partial v}{\partial y}+\frac{\partial w}{\partial z}=0$

Momentum denklemi

x momentum denklemi

$\rho\left(u \frac{\partial u}{\partial x}+v \frac{\partial u}{\partial y}+w \frac{\partial u}{\partial z}\right)=-\frac{\partial p}{d x}+\mu\left(\frac{\partial^{2} u}{\partial x^{2}}+\frac{\partial^{2} u}{\partial y^{2}}+\frac{\partial^{2} u}{\partial z^{2}}\right)$

y momentum denklemi

$\rho\left(u \frac{\partial v}{\partial x}+v \frac{\partial v}{\partial y}+w \frac{\partial v}{\partial z}\right)=-\frac{\partial p}{\partial y}+\mu\left(\frac{\partial^{2} v}{\partial x^{2}}+\frac{\partial^{2} v}{\partial y^{2}}+\frac{\partial^{2} v}{\partial z^{2}}\right)$

z momentum denklemi

$\rho\left(u \frac{\partial w}{\partial x}+v \frac{\partial w}{\partial y}+w \frac{\partial w}{\partial z}\right)=-\frac{\partial p}{\partial z}+\mu\left(\frac{\partial^{2} w}{\partial x^{2}}+\frac{\partial^{2} w}{\partial y^{2}}+\frac{\partial^{2} w}{\partial z^{2}}\right)$

Enerji denklemi

$u \frac{\partial T}{\partial x}+v \frac{\partial T}{\partial y}+w \frac{\partial T}{\partial z}=\left(\frac{k}{\rho c_{p}}\right)\left(\frac{\partial^{2} T}{\partial x^{2}}+\frac{\partial^{2} T}{\partial y^{2}}+\frac{\partial^{2} T}{\partial z^{2}}\right)$

$\mathrm{Bu}$ denklemlerde, $\rho$ yoğunluğu, $\mu$ dinamik viskoziteyi, $\mathrm{p}$ basıncı, $\mathrm{k}$ 1sıl iletkenliği, $\mathrm{T}$ sıcaklığı, $c_{p}$ özgül ısıyı ve u, v, w sırasıyla x, y ve z yönlerindeki hızları göstermektedir.

Kullanılan standart k- $\varepsilon$ türbülans modelinde, türbülans kinetik enerji, $\in$ yayılma oranı ve $\phi$ viskoz dağılım terimidir. 
Daimi akış türbülans kinetik enerji denklemi

$$
\frac{\partial\left(\rho u k^{\prime}\right)}{\partial x}+\frac{\partial\left(\rho v k^{\prime}\right)}{\partial y}+\frac{\partial\left(\rho w k^{\prime}\right)}{\partial z}=\frac{\partial}{\partial x}\left(\frac{\mu_{t}}{\sigma_{k}} \frac{\partial k^{\prime}}{\partial x}\right)+\frac{\partial}{\partial y}\left(\frac{\mu_{t}}{\sigma_{k}} \frac{\partial k^{\prime}}{\partial y}\right)+\frac{\partial}{\partial z}\left(\frac{\mu_{t}}{\sigma_{k}} \frac{\partial k^{\prime}}{\partial z}\right)+\mu_{t} \phi-\rho \varepsilon
$$

Türbülans viskozitesi

$$
\mu_{t}=C_{\mu} \rho \frac{k^{\prime 2}}{\varepsilon}
$$

Türbülans kinetik enerji

$$
k^{\prime}=\frac{1}{2}\left(\overline{u^{\prime 2}}+\overline{v^{\prime 2}}+\overline{w^{\prime 2}}\right)
$$

Viskoz dağ 1 lım terimi

$$
\phi=2 \mu\left[\left(\frac{\partial u}{\partial x}\right)^{2}+\left(\frac{\partial v}{\partial y}\right)^{2}+\left(\frac{\partial w}{\partial z}\right)^{2}\right]+\mu\left[\left(\frac{\partial v}{\partial x}+\frac{\partial u}{\partial y}\right)^{2}+\left(\frac{\partial w}{\partial y}+\frac{\partial v}{\partial z}\right)^{2}+\left(\frac{\partial u}{\partial z}+\frac{\partial w}{\partial x}\right)^{2}\right]
$$

Türbülans kinetik enerji kaybolma denklemi

$$
\frac{\partial(\rho u \varepsilon)}{\partial x}+\frac{\partial(\rho v \varepsilon)}{\partial y}+\frac{\partial(\rho w \varepsilon)}{\partial z}=\frac{\partial}{\partial x}\left(\frac{\mu_{t}}{\sigma_{\varepsilon}} \frac{\partial \varepsilon}{\partial x}\right)+\frac{\partial}{\partial y}\left(\frac{\mu_{t}}{\sigma_{\varepsilon}} \frac{\partial \varepsilon}{\partial y}\right)+\frac{\partial}{\partial z}\left(\frac{\mu_{t}}{\sigma_{\varepsilon}} \frac{\partial \varepsilon}{\partial z}\right)+C_{1 \varepsilon} \mu_{t} \frac{\varepsilon}{k^{\prime}} \phi-C_{2 \varepsilon} \rho \frac{\varepsilon^{2}}{k^{\prime}}
$$

Model sabitleri $C_{\mu}, C_{1 \varepsilon}, C_{2 \varepsilon}, \sigma_{k}$ ve $\sigma_{\varepsilon}$ standart k- $\varepsilon$ türbülans modeli için kullanılan varsayılan değerlerdir ([4], [6], [8]). Bu sabitlerin değerlerine, çok çeşitli türbülanslı akışlar için çok sayıda veri uydurma yinelemesi ile ulaşılmıştır. Bu sabitler şu şekilde$\operatorname{dir} ; C_{\mu}=0.09, C_{1 \varepsilon}=1.44, C_{2 \varepsilon}=1.92, \sigma_{k}=1$ ve $\sigma_{\varepsilon}=1.3$.

Şekil 10'da çatısız bina modeli için binanın üst, ön ve özellikle de arka bölgelerinde oluşan akış yapısını araştırmak amacıyla zaman ortalama $<\mathrm{u}>$ hız bileşeninin kanal yüksekliğine bağlı olarak değişimi boyutsuz şekilde deneysel ve sayısal olarak karşılaştırılmıştır. Grafik, tek bina modeli için $\mathrm{z} / \mathrm{H}=0.5$ simetri yüzeyi için çizilmiştir. Grafik analiz edildiğinde, bazı bölgelerde deneysel ve sayısal sonuçlar arasında \% 3'lük bir fark olduğu saptanmıştır. Bu farklılığın sebebinin özellikle bina üst kısımlarında akışın değişkenlik göstermesinden kaynaklandığı düşünülmektedir. Bina arka bölgelerinde ayrılmış akış bölgeleri oluşmakta ve ayrılmış akış, $\mathrm{z} / \mathrm{H}=0.45$ profilinden başlayarak $\mathrm{z} / \mathrm{H}=0.75$ profiline kadar azalarak devam etmektedir. Bununla birlikte, sayısal ve deneysel sonuçlar arasında en fazla \% 3'e kadar görülen sapmanın deneysel sonuçların kabul edilebilir doğrulukta, makul ve uygun olduğunu düşündürmektedir. Ayrıca, grafik üzerinde gösterilen bina skalasız çizilmiştir. 


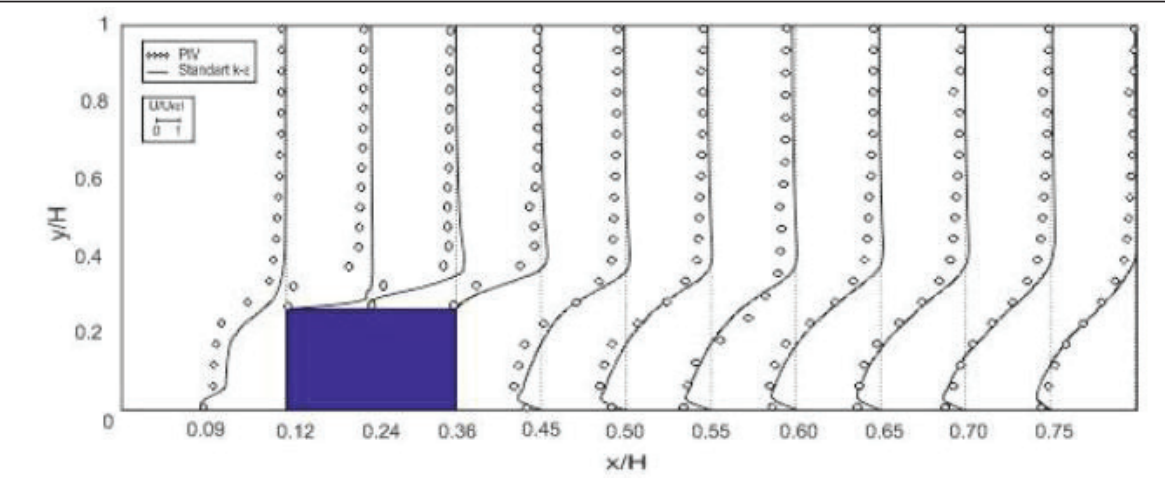

Şekil 10. Çatısız Tek Bina Modeli Için z/H=0.5 Simetri Yüzeyinde $\frac{\mathrm{u}}{\mathrm{U}_{\infty}}$ Hız Profillerinin Deneysel

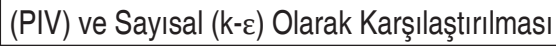

\section{GENEL SONUÇLAR}

Bu çalışmada, bina merkezleri arası mesafenin $7.5 \mathrm{~cm}$ ve $10 \mathrm{~cm}$ olduğ $5 \mathrm{~cm} \times 5 \mathrm{~cm} \times 5 \mathrm{~cm}$ boyutlarında çapraz olarak yerleştirilmiş üç adet çatısız bina modelinin etrafindaki akış yapıları deneysel olarak Parçacık Görüntülemeli Hız tekniği (PIV) kullanılarak incelenmiştir. Ayrıca, çatısız $5 \mathrm{~cm} \times 5 \mathrm{~cm} \times 5 \mathrm{~cm}$ boyutlarındaki bir binanın etrafındaki akış analizi sayısal olarak üç boyutlu, daimi, k-ع türbülans modeli ile ANSYS Fluent bilgisayar programı kullanılarak yapılmış ve ulaşılan sayısal sonuçlar deneysel sonuçlarla karşılaştırılmıştır. Böylece, aşağıda belirtilen sonuçlara ulaşılmıştır.

1. Her iki bina arası mesafe için binaların yan kısımlarında ve bina arkalarındaki girdaplar incelendiğinde birbirlerine simetrik bir yapıda olmadıkları görülmektedir. Bu duruma, gelen akışın binaların köşe ve kenarlarından ayrılmasıyla yapıların önünde ve yan duvarları boyunca ve arka kısmında oluşan ters akış bölgeleri sebep olmaktadır. Bu durumun sonucu olarak da girdapların olduğu kısımlarda vektör yoğunluklarında azalmalar görülmektedir ki bu sonuç da buralarda akış hızının azaldığını kanıtlamaktadır. Bu nedenle, binalar konumlandırılırken etraflarındaki rüzgar akışı dikkate alınarak tasarlanmalıdır.

2. Ters ve serbest akış bölgelerini ayıran karışım bölgesinde türbülans büyüklükleri artış göstermektedir. Bu durum, binalara etkiyen rüzgar kuvvetlerini artırmaktadır.

3. Binalar arası mesafenin $10 \mathrm{~cm}$ olduğu durumda, $7.5 \mathrm{~cm}$ olduğu duruma göre jet akış alanı genişlemiş ve buna bağlı olarak bina arka bölgelerinde farklı girdap yapıları oluşmuştur. Ayrıca arka kısma yerleştirilmiş binanın yan bölgelerinde oluşan girdap yapıları küçülerek değişime uğramıştır.

4. Binaların akış ortamında bir arada bulunmaları, binalar etrafındaki akış yapılarının 
birbirleriyle karşılıklı etkileşim içerisinde bulunmalarına sebep olmaktadır. Bununla birlikte, binalar arası mesafeye bağlı olarak binalar arasında oluşan jet akış nedeniyle özellikle binalara yakın bölgelerde hızlar yükselmiş ve binalar arkasındaki girdap büyüklükleri değişiklik göstermiştir.

5. Binalar arası mesafenin $7.5 \mathrm{~cm}$ olduğu durumla kıyaslandığında, mesafenin $10 \mathrm{~cm}$ olması durumunda jet akış bölgesinin oldukça genişlediği ve çapraz olarak yerleştirilen bina çevresindeki ayrılmış akış bölgesinin küçüldüğü görülmektedir.

6. Deneysel ve sayısal sonuçlar arasında en fazla \% 3' lük bir fark olduğu saptanmıştır. $\mathrm{Bu}$ farklılığa da bina üst kısımlarında oluşan girdaplar nedeniyle akışın değişkenlik göstermesinin sebep olduğu düşünülmektedir. Ayrıca, bina arka bölgelerinde ayrılmış akış bölgelerinin oluştuğu ve ayrılmış akışın $\mathrm{z} / \mathrm{H}=0.45$ profilinden başlayarak $\mathrm{z} / \mathrm{H}=$ 0.75 profiline kadar azalarak devam ettiği belirlenmiştir.

Sonuç olarak, binalar etrafında oluşan akış yapısına ve binalar üzerine etkiyen rüzgar kuvvetlerine, gelen rüzgarın yönü ve şiddetinin yanı sıra binaların yerleşim şeklinin ve binalar arası mesafenin etkili olduğu görülmektedir.

\section{SEMBOLLER}

$\begin{array}{ll}\psi & \text { Akım çizgisi } \\ \mathrm{V} & \text { Vektörel hız dağılımı } \\ \mathrm{H} & \text { Bina yüksekliği, }[\mathrm{cm}] \\ \mathrm{X} & \text { Girdap uzunluğu, }[\mathrm{cm}] \\ \mathrm{Sa} & \text { Bina ön durma noktası } \\ \mathrm{Sn} & \text { Durma noktası } \\ \mathrm{F} & \text { Girdap } \\ \mathrm{k} & \text { Isı iletim katsayısı, }[\mathrm{W} / \mathrm{m} \mathrm{K}] \\ \mathrm{k}^{\prime} & \text { Türbülans kinetik enerji, }[\mathrm{m} 2 / \mathrm{s} 2] \\ \mathrm{u}^{\prime}, \mathrm{v}^{\prime}, \mathrm{w}^{\prime} & \mathrm{x}, \mathrm{y}, \mathrm{z} \text { yönlerindeki hız bileşenleri, }[\mathrm{m} / \mathrm{s}] \\ \mathrm{u}^{\prime}, \mathrm{v}^{\prime}, \mathrm{w} & \mathrm{x}, \mathrm{y}, \mathrm{z} \text { yönlerindeki dalgalanan hız bileşenleri, }[\mathrm{m} / \mathrm{s}] \\ \mathrm{x}, \mathrm{y}, \mathrm{z} & \text { Kartezyen koordinatlar, }[\mathrm{m}] \\ \varepsilon & \text { Türbülans dağılım oranı, }\left[\mathrm{m}^{2} / \mathrm{s}^{3}\right] \\ \mu & \text { Dinamik viskozite, }[\mathrm{kg} / \mathrm{s} \mathrm{m}] \\ \mu_{\mathrm{t}} & \text { Türbülans viskozitesi, }[\mathrm{kg} / \mathrm{s} \mathrm{m}] \\ \rho & \text { Yoğunluk, }\left[\mathrm{kg} / \mathrm{m}^{3}\right] \\ \phi & \text { Viskoz dağılım terimi, }\left[\mathrm{m}^{2} / \mathrm{s}^{3}\right] \\ v & \text { Kinematik viskozite, }\left[\mathrm{m}^{2} / \mathrm{s}\right]\end{array}$




\section{TEŞEKKÜR}

Bu çalışma, Sivas Cumhuriyet Üniversitesi Bilimsel Araştırma Projeleri (CÜBAP) birimi tarafından M-531 proje numarası ile desteklenmiştir.

\section{KAYNAKÇA}

1. Gölbaşı, D., Buyruk, E., Şahin, B., Karabulut, K. 2017. "Değiş̧ik Bina Modelleri için Akış Alanlarının Deneysel ve Sayısal Olarak İncelenmesi”, Tesisat Mühendisliği, cilt 6, say1 162 s. 32-47. https://www.mmo.org.tr/istanbul-merkez/tesisat-muhendisligi-dergisi/eylul-ekim-2017-sayi-161.

2. Tutar, M., Oguz, G. 2002. "Large Eddy Simulation of Wind Flow Around Parallel Buildings with Varying Configurations, Fluid Dynamics Research", cilt 31, say1 5-6, s. 289-315. https://doi.org/10.1016/S0169-5983(02)00127-2.

3. Blocken, B., Dezsö, G., Beeck, J., Carmeliet, J. 2009. “The Mutual Influence of Two Buildings on Their Wind Driven Rain Exposure and Comments on the Obstruction Factor", Journal of Wind Engineering and Industrial Aerodynamics, cilt 97, say1 5-6, s. 180-196. https://doi.org/10.1016/j.jweia.2009.06.003.

4. Gölbaşı, D., Buyruk, E., Karabulut, K. 2018. "Experimental and Numerical Research of the Flow Features Around the Building Pairs with Different Types", Cumhuriyet Science Journal, cilt 39, say1 4, s. 1089-1106. https://doi.org/10.17776/ csj.393304.

5. Gölbaşı, D., Buyruk, E., Şahin, B., Karabulut, K., Kılınc, F. 2017. "Bina Yüksekliği Değişiminin Akış Yapıları Üzerindeki Etkisinin Deneysel ve Sayısal Olarak İncelenmesi”, ULİBTK'17 21. Ulusal Isı Bilimi ve Tekniği Kongresi, 13-16 Eylül 2017, Çorum. https://www.researchgate.net/publication/327051437_Bina_Yuksekligi_Degisiminin_Akis_Yapilari_Uzerindeki_Etkisinin_Deneysel_ve_Sayisal_Olarak_Incelenmesi.

6. Daemei, A. B., Eghbali, S. R. 2019. "Study on Aerodynamic Shape Optimization of Tall Buildings Using Architectural Modifications In Order to Reduce Wake Region", Wind and Structures, cilt 29, say1 2, s. 139-147. http://dx.doi.org/10.12989/ was.2019.29.2.139.

7. Wheatley, C., Baumann, M., Gutierrez, S. M. 2020. "Aerodynamic Mitigation of Origami-Inspired Building Structures Subjected to Hurricane Wind Loads”, Proc. SPIE 11377, Behavior and Mechanics of Multifunctional Materials IX, 1137708, 18 Mayss 2020, Online. https://www.spiedigitallibrary.org/conference-proceedingsof-spie/11377/1137708/Aerodynamic-mitigation-of-origami-inspired-buildingstructures-subjected-to hurricane/10.1117/12.2558972.short? $\mathrm{SSO}=1$.

8. Yan, B., Li, Q. S. 2016. "Wind Tunnel Study of Interference Effects Between Twin Super-Tall Buildings with Aerodynamic Modifications", Journal of Wind Engine- 
ering and Industrial Aerodynamics, cilt 156, s. 129-145. https://doi.org/10.1016/j. jweia.2016.08.001.

9. Gölbaşı, D. 2015. "Yerleşim Alanları İçerisinde Farklı Geometrilere Sahip Yapılar Üzerinde Isı Transferi ve Akış Yapılarının Deneysel ve Teorik Olarak İncelenmesi”, Doktora Tezi, Sivas Cumhuriyet Üniversitesi Fen Bilimleri Enstitüsü, Sivas. https:// tez.yok.gov.tr/UlusalTezMerkezi/tezSorguSonucYeni.jsp.

10. Gousseau, P., Blocken, B., Stathopoulos, T., Heijst, G. J. F. 2011. “CFD Simulation of Near Field Pollutant Dispersion on A High Resolution Grid: A Case Study by Les and Rans for A Building Group in Downtown Montreal", Atmospheric Environment, cilt 45, say1 2, s. 428-438. https://doi.org/10.1016/j.atmosenv.2010.09.065.

11. FLUENT, 2003. "Fluent 6.1 User's Guide”, Fluent Inc., Lebanon. http://jullio.pe.kr/ fluent6.1/help/pdf/ug/fl61ug.pdf. 